\title{
SU8 Derived Glassy Carbon for Lithium Ion Batteries
}

\author{
Swati Sharma, ${ }^{1 *}$ Amirhossein Khalajhedayati, ${ }^{2,3}$ Timothy J. Rupert, ${ }^{2,3}$ Marc J. Madou ${ }^{1,3,4}$ \\ ${ }^{I}$ Materials and Manufacturing Technology, University of California Irvine, Irvine, CA, USA, 92697 \\ ${ }^{2}$ Department of Chemical Engineering and Materials Science, University of California Irvine, Irvine, CA, \\ USA, 92697 \\ ${ }^{3}$ Department of Mechanical and Aerospace Engineering, University of California Irvine, Irvine, CA, USA, \\ 92697 \\ ${ }^{4}$ Department of Biomedical Engineering, University of California Irvine, Irvine, CA, USA, 92697
}

\begin{abstract}
Glassy carbon (GC) is an amorphous yet $s p^{2}$ rich allotrope of carbon that has received a lot of attention as an electrode material in $\mathrm{Li}$ ion batteries. In this contribution we explore the microstructural, thermal and mechanical properties of GC derived from the pyrolysis of lithographically patterned SU8, which is the most common precursor polymer in CMEMS (carbon microelectromechanical systems). Microstructural characterization of CMEMS carbon in terms of interlayer separation of the characteristic turbostratic (randomly arranged) graphene layers, which influences the mechanism of lithiation and accompanying swelling and heating during battery operation, is carried out employing electron microscopy. Mechanical properties of the thus obtained GC are also investigated using nanoindentation for films, and microcompression tests for micropillars. Rapid heating/cooling of the miniaturized GC structures due to their relatively large surface area is demonstrated by Joule heating of a carbon nanowire (CNW) suspended onto CMEMS walls.
\end{abstract}

\section{Introduction}

SU8 is the commercial name for an epoxy based phenol-formaldehyde type negative tone photoresist whose chemical structure is illustrated in Figure 1(A). SU8 has several attributes that make it suitable for the fabrication of high aspect ratio MEMS structures through photolithography. It is photosensitive in the $300-400 \mathrm{~nm}$ range, biocompatible, and features high thermal and chemical stability after cross-linking and development(1). SU8 can also be utilized for the fabrication of carbon micro- and nanoelectromechanical systems i.e., carbon MEMS (CMEMS) and carbon NEMS (CNEMS) since it yields a low-porosity highly graphitic glassy carbon (GC) when pyrolyzed at $900^{\circ} \mathrm{C}$ with a $<5^{\circ} \mathrm{C} /$ minute temperature ramp rate(2). The network of long aromatic molecular chains in SU8 [Figure 1(A)] with a carbon backbone and epoxy functional groups in its side chains facilitates three-dimensional cross-linking, and upon pyrolysis a large number of $\mathrm{C}-\mathrm{C}$ bonds are rapidly formed to yield a carbon material with embedded graphite crystallites composed of $s p^{2}$ hybridized carbon atoms. 
Carbon derived from the pyrolysis of any precursor polymer exhibits glass-like physical properties. Unlike most amorphous materials GC features very good electrical conductivity owing to electron transport by free movement of electrons in the $s p^{2}$ hybridized carbon. In addition, the low density, corrosion resistance, low thermal expansion, low elasticity, and high purity of GC make it a suitable material for electrodes(3). GC also exhibits a wide electrochemical window and may substitute more expensive noble metals commonly employed for the fabrication of miniaturized electrodes. CMEMS and CNEMS technology have enabled the fabrication of a wide variety of miniature, on-chip GC electrode designs employing lithographic patterning of photoresists (mainly SU8) followed by pyrolysis, resulting in electrochemical devices with novel geometries and unprecedented sensitivities(4).

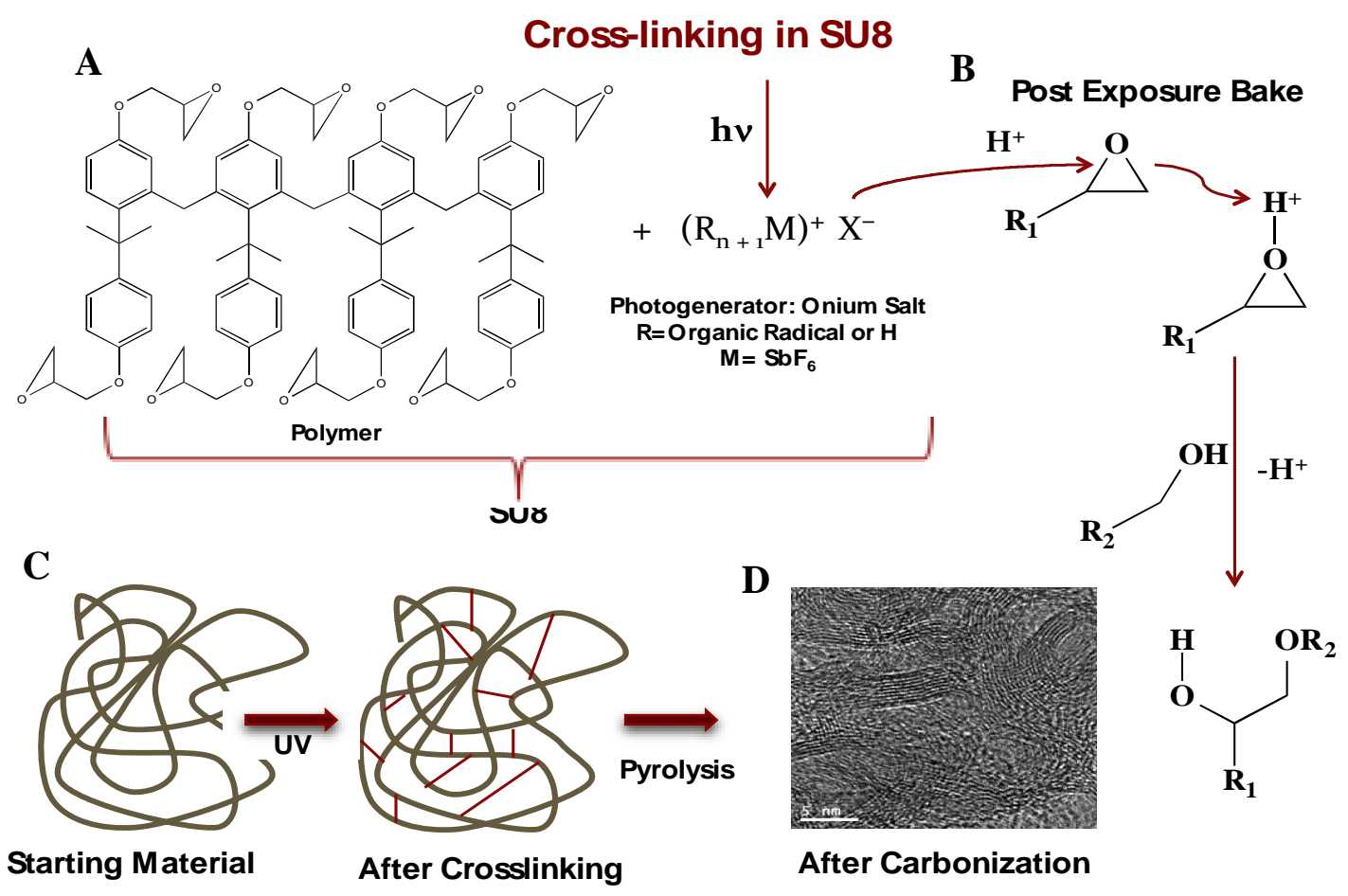

Figure 1: (A) Chemical structure of SU8, (B) Cross-linking of SU8 polymer chains on UV exposure followed by post exposure bake at $95^{\circ} \mathrm{C}$, (C) schematic representation of $3 \mathrm{D}$ crosslinking in SU8, and (D) microstructure of SU8 derived glassy carbon on pyrolysis at $900^{\circ} \mathrm{C}$.

One of the most rapidly growing fields in terms of electrochemical applications of GC electrodes is in Li ion batteries(5-7). Recently, Li ion batteries with carbon based anodes have become one of the most popular energy storage systems for portable electronic devices such as mobile phones, cardiac pacemakers, hearing aids, smart cards, and remote sensors(5). In the near future these rechargeable batteries are also expected to provide energy for zero emission vehicles(8).

Li is a powerful reducing agent, which can facilitate the fabrication of high voltage and highenergy batteries when associated with strong oxidants(8). However, the deposition of dendritic 
lithium metal during the charging of the battery limits its applications and raises safety concerns. This problem can be avoided by introducing a host structure as a matrix that can reversibly absorb and release $\mathrm{Li}$ ions (through intercalation) at low electrochemical potentials(9). The lifetime and usability of a lithium-based battery can be drastically improved this way. However, several lithiation cycles may lead to heating and mechanical failure of the host structure(8). Hard, electrically conducting materials with a high melting temperature and sufficient space for the movement of $\mathrm{Li}$ ions are therefore preferred for this purpose.

As clear from Figure 1(B), the SU8 derived GC microstructure features entangled ribbon-like geometries of $s p^{2}$ type carbon (ribbon thickness in the 2-5 $\mathrm{nm}$ range), and the spaces between these ribbons are either voids or filled with amorphous carbon. The ribbons are composed of a few graphene layers stacked onto each other in a random arrangement, i.e., not arranged in a graphite-like hexagonal closed packed (HCP) crystal form. This random orientation of graphene layers is known as a turbostratic arrangement, which results in a slightly wider separation between the two graphene layers than in graphite. This turbostratic form of GC may render it more suitable for intercalation, i.e., Li ions can avail more space for insertion/ removal, as a result there also may be less associated swelling/ heating compared to $\mathrm{HCP}$ graphite.

With the advancements in CMEMS technology, miniature GC electrodes have gained popularity in electrochemical applications(10). GC patterns on $\mathrm{Si}$ chips(2) or plastic substrates(11) have opened up an entirely new research field of developing CMEMS based advanced Li ion batteries(12). For example, an array of GC micropillars on a Si substrate was successfully evaluated for battery applications by our group (5).

As postulated by Wang et al.,(12) the advantages that batteries based on 3D carbon micropillars have over those based on 2D thin films are (i) higher energy density, (ii) higher capacity, (iii) faster charging and, (iv) reconfigurability for load leveling (smart battery concept). We believe also that these CMEMS electrodes will be more resistant against thermal cracking (low Biot number) and exhibit improved mechanical durability. Three important features of an ideal lithium ion battery electrode material are: (i) allows for the easy implementation of any desired electrode shape, (ii) withstands many Li insertion/ removal cycles without mechanical failure, and (iii) is able to withstand high temperatures. The CMEMS fabrication process where the starting material is a carbonizable and patternable polymer ensures the ease of fabrication in any geometry, but, as suggested above, not much is known yet about the thermal and mechanical properties of CMEMS and CNEMS derived GC.

In this contribution the microstructure and interlayer separation in CMEMS GC, which affect both mechanical and thermal properties, are explored employing High Resolution Transmission Electron Microscopy (HRTEM). High temperature tolerance and mechanical strength of CMEMS structures are investigated using Joule heating and hardness test, respectively. More specifically the hardness tests for both GC films and micropillars were carried out using nanoindentation. Nanoindentation has gained a lot of attention as a technique for determining the mechanical properties of metallic, organic, inorganic and metal-organic materials(13-14). With this technique one can investigate the mechanical properties of thin (by indenting their surface) as well as perform microcompression tests for pillars (by compressing the pillar with a nanoindenter tip large in size compared to the pillar diameter). In the recent past nanoindentation has also been used for investigating the properties of viscoelastic polymeric materials, and experimental conditions such as tip geometry and indentation depths for these relatively soft 
materials have been extensively researched(14). By studying their mechanical behavior, we aim to better understand the chemical and structural changes in polymeric SU8- carbon intermediate materials obtained during pyrolysis of photoligraphically patterned SU8. Details of the CMEMS fabrication process and the nanoindentation induced mechanical deformation are discussed below.

\section{Materials and Methods}

$\underline{\text { Photolithography and Pyrolysis }}$

CMEMS designs for all tests described here were first photopatterned in SU8 employing standard UV photolithography. These designs include micropillars, films and SU8 microwalls. For the heat resistance tests a SU8 nanowire was suspended onto SU8 microwalls using electrospinning. This assembly was then carbonized in an inert atmosphere to yield the suspended carbon nanowires for the Joule heating experiments.

In the photolithography step preceding pyrolysis, an SU8 formulation with the optimal viscosity for the intended application was spin-coated onto the desired substrate. The spin coater's rotation speed, resist viscosity and resist concentration was fine tuned to control the thickness of the resulting resist film. To evaporate the solvent from the polymer the resist film is then baked in two steps: first the sample is heated to $65^{\circ} \mathrm{C}$ and then to $95^{\circ} \mathrm{C}$ in order to ensure slow and uniform solvent evaporation from the resist film. After the pre- exposure bake the resist film is exposed to UV light through a mask. In this case of a negative photoresist such as SU8, the UV light activates the photoinitiators in the polymer, which leads to cross-linking when the film is baked for a second time in the post-exposure bake (Figure 1A,B). The cross-linked areas become harder and more difficult to dissolve than the parts that were not exposed to UV (Figure 1C). The entire pattern is then immersed in a developer solution (supplied by the manufacturer), which selectively dissolves away the uncrosslinked parts of the polymer. The resulting patterns are then washed with isopropyl alcohol and dried by gently blowing $\mathrm{N}_{2}$ on them. The samples are pyrolyzed after photopatterning to convert into carbon (Figure 1D). The standard pyrolysis used in CMEMS and CNEMS manufacturing process is carried out at a temperature ramp rate below $10^{\circ} \mathrm{C} /$ minute to the final temperature of $900^{\circ} \mathrm{C}$.

Establishing the correct UV exposure dose for a given SU8 film thickness is of the utmost importance since both under- and over-exposed films lead to fabrication failures. An underexposed design results in incomplete cross-linking at the base of the film and hence weak adhesion to the substrate and such patterns are usually washed away during development. On the other hand, an over-exposed design leads to T-topping, yielding T-shaped features(15).

\section{HRTEM of SU8 derived GC}

To prepare HRTEM samples thick films of SU8 $(5 \mathrm{~mm}$ x $5 \mathrm{~mm}$ x $200 \mu \mathrm{m})$ were patterned onto a Si wafer and pyrolyzed using the method described above. These thick SU8 films usually crack during pyrolysis because of the stresses generated by the shrinkage accompanying this process. One such film was carefully peeled-off from the substrate, and converted into a fine powder. This powdered sample was analyzed in a JEM-2100F (Cs) HRTEM (JEOL, Japan) set-up. In addition to micrographs, selected area electron diffraction (SAED) patterns with intensity profiles of the graphite crystallites in GC at various spatial positions were taken. 


\section{$\underline{\text { Mechanical Strength Measurements }}$}

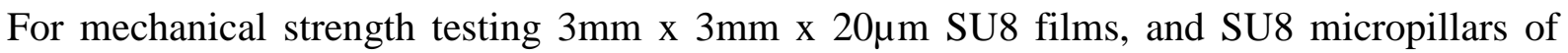
$80 \mu \mathrm{m}$ height and $15 \mu \mathrm{m}$ diameter were fabricated on a p- type Si wafer [(100) orientation] employing photolithography. These samples were pyrolyzed in a high temperature furnace (Lindberg Nanofurnace, USA) at $900^{\circ} \mathrm{C}$ under 0.2 liter/ minute flow of $\mathrm{N}_{2}$. After pyrolysis the thickness of the films had shrunk to $\sim 10 \mu \mathrm{m}$, and the micropillar dimensions were now $30 \mu \mathrm{m}$ in height and $7.5 \mu \mathrm{m}$ in diameter. Nanoindentation tests were carried out to calculate hardness and the Young's modulus of the CMEMS film samples, and the strength of micropillars. An Agilent Nano Indenter G200 system employing a diamond Berkovich-type indenter tip with DCM II head was used at a constant strain rate of 0.05/s. The area function and load frame compliance was calibrated on a standard fused silica specimen at the same load level. The load-displacement data for a $1.5 \mu \mathrm{m}$ indentation depth were collected at 49 different points spread over the entire geometric surface of each sample. A 5 seconds hold was implemented at maximum depth to reduce the viscoelastic effect on the unloading slope(14).

Microcompression tests were performed on the CMEMS pillars using the same Nanoindenter but with a triangular flat diamond tip with sides of $31 \mu \mathrm{m}$. The compression tests were carried out under a constant displacement rate of $10 \mathrm{~nm} . \mathrm{s}^{-1}$. After the compression, the data were corrected for indentation into the substrate using the Sneddon equation(14). The height and diameter of each pillar was measured using SEM images, and the stress-strain data were calculated by assuming uniform plastic deformation.

\section{Thermal Properties of SU8 Derived Carbon Nanowires under Joule Heating}

CMEMS patterns consisting of two parallel SU8 walls $(20 \mu \mathrm{m}$ wide, $10 \mu \mathrm{m}$ high and $20 \mu \mathrm{m}$ apart) were fabricated employing UV photolithography. A SU8 nanowire ( 200nm thick, 20 $\mu \mathrm{m}$ long before pyrolysis) was then suspended by electrospinning between these SU8 walls, which, after pyrolysis, served as contacts to the suspended nanowire. Details of the fabrication process are reported elsewhere(16). After pyrolysis the CMEMS structure was connected to a power supply and the suspended nanowire was heated employing Joule heating(17).

\section{Results and Discussion}

\section{$\underline{\text { SEM Characterization }}$}

Typical SEM micrographs of GC micropillars and CNWs suspended on CMEMS walls are shown in Figure $2(\mathrm{~A}-\mathrm{C})$. The average height and diameter of the pillars were $30 \mu \mathrm{m}$ and $7.5 \mu \mathrm{m}$ respectively (aspect ratio: 4 ).

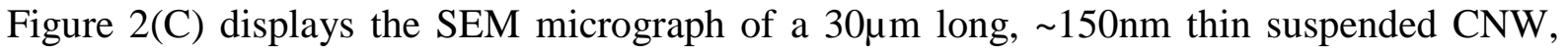
broken at its center after a current of $0.5 \mathrm{~mA}$ was passed through it. Theoretically, even with a lower current of $0.1 \mathrm{~mA}$ the center of a CNW of this dimension should reach $4500^{\circ} \mathrm{C}$ and break long before this temperature is reached, since the sublimation temperature of $\mathrm{GC}$ is $3600^{\circ} \mathrm{C}(17)$. However, we observed that the CNWs show the first signs of damage only when a $0.5 \mathrm{~mA}$ current was passed through it for at least 15 minutes. Miniaturized (micro and nano scale) GC structures such as CMEMS and CNEMS do not reach these theoretically projected higher temperatures upon Joule heating owing to their very large surface area. These data suggest that 
incorporating micro and nano GC CMEMS/CNEMS features might be very useful for fast heating and cooling of battery electrodes.
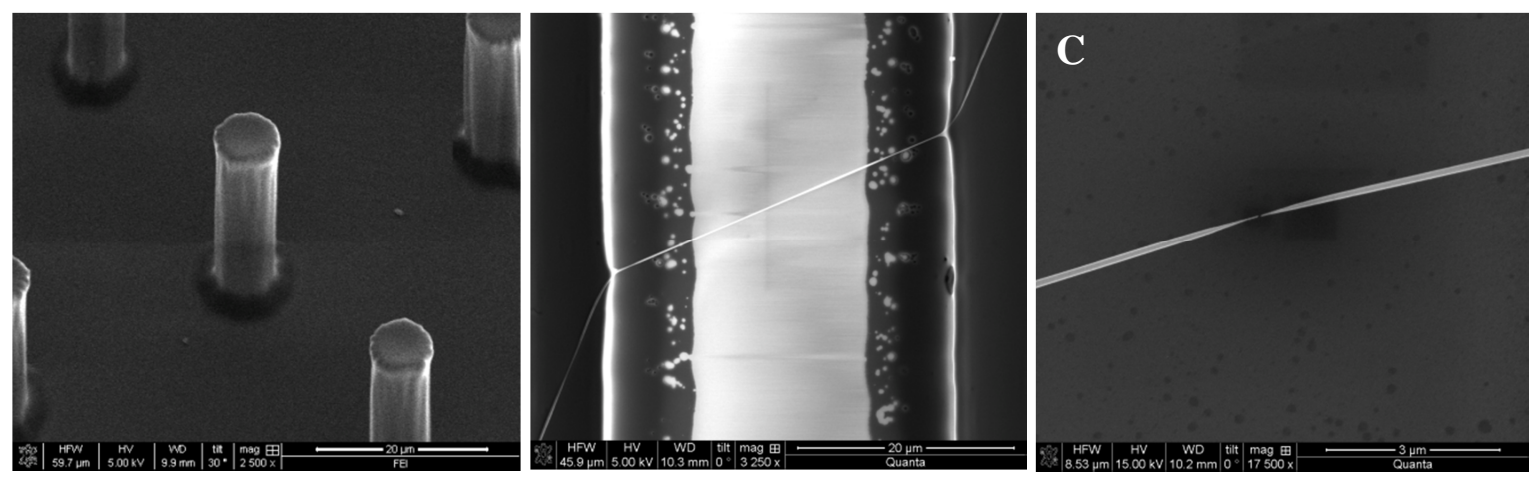

Figure 2: Scanning Electron Micrographs of (A) glassy carbon micropillars, (B) Carbon Nanowire suspended onto carbon walls, and (C) damage at the center of a $150 \mathrm{~nm}$ thin, $30 \mu \mathrm{m}$ long carbon nanowire on passing $0.5 \mathrm{~mA}$ current.

The suspended CNW structures described here also constitute the core of a new type of nano gas sensor fabrication method where the Joule heating of the CNW up to high temperatures without any structural damage is utilized for the selective area chemical vapor deposition (CVD) of gas sensitive materials. The details of this application are reported elsewhere(17).

\section{$\underline{\text { Microstructural Characterization }}$}

In Figure 3(A-E) we illustrate an HRTEM micrograph (Figure 3A), selected area electron diffraction (SAED) patterns (Figures 3B,C), and intensity profiles (Figures 3D,E) used to derive the interlayer separation in the graphite crystallites present in CMEMS GC. In case of a graphite $\mathrm{HCP}$ crystal the interlayer separation is $0.335 \mathrm{~nm},(18)$ but in GC it is usually $>0.34 \mathrm{~nm}$. Here we find 0.36 and $0.353 \mathrm{~nm}$ (Figures $3 \mathrm{D}$ and E respectively).

During carbonization of a crosslinked polymer, the heteroatoms (such as $\mathrm{O}, \mathrm{H}$ or $\mathrm{N}$ present in the polymer) are released and new $\mathrm{C}$ - $\mathrm{C}$ bonds are formed. It is expected that in the case of aromatic polymers (such as SU8), the entangled long molecular chains (backbones) are preferentially converted into graphene sheets over the material in the side chains, which are comparatively more oxygen rich due to the epoxy groups. The material surrounding the backbone chains is hence more likely to be converted either to amorphous carbon, or to just leave voids/ pores(19). A stack of a few such graphene layers that is sufficiently long and interconnected with other similar stack-structures forms the three-dimensional network of $s p^{2}$ carbon ribbons (HRTEM micrograph: Figure 3A) typical for glassy carbon.

The graphene sheets in these ribbons are not arranged in the ABABA fashion, characteristic of HCP graphite, but are randomly stacked on top of each other. This turbostratic layer arrangement in the GC microstructure results in a larger separation between two adjacent layers compared to graphite. During intercalation, Li ions are inserted between graphite sheets, which results in swelling of the electrode owing to the fact that $\mathrm{Li}$ insertion increases the interlayer distance in graphite. Given that CMEMS GC has a wider gap between adjacent $s p^{2}$ carbon sheets less swelling (and subsequent shrinking) is expected in the case of these electrodes compared to graphite electrodes. Along this same line, in a theoretical study, Xing et al.(20) suggested that 
the microstructure of hard carbons derived from organic precursors such as GC composed of graphene sheets with a possibility of pores features a larger reversible Li ion capacity.
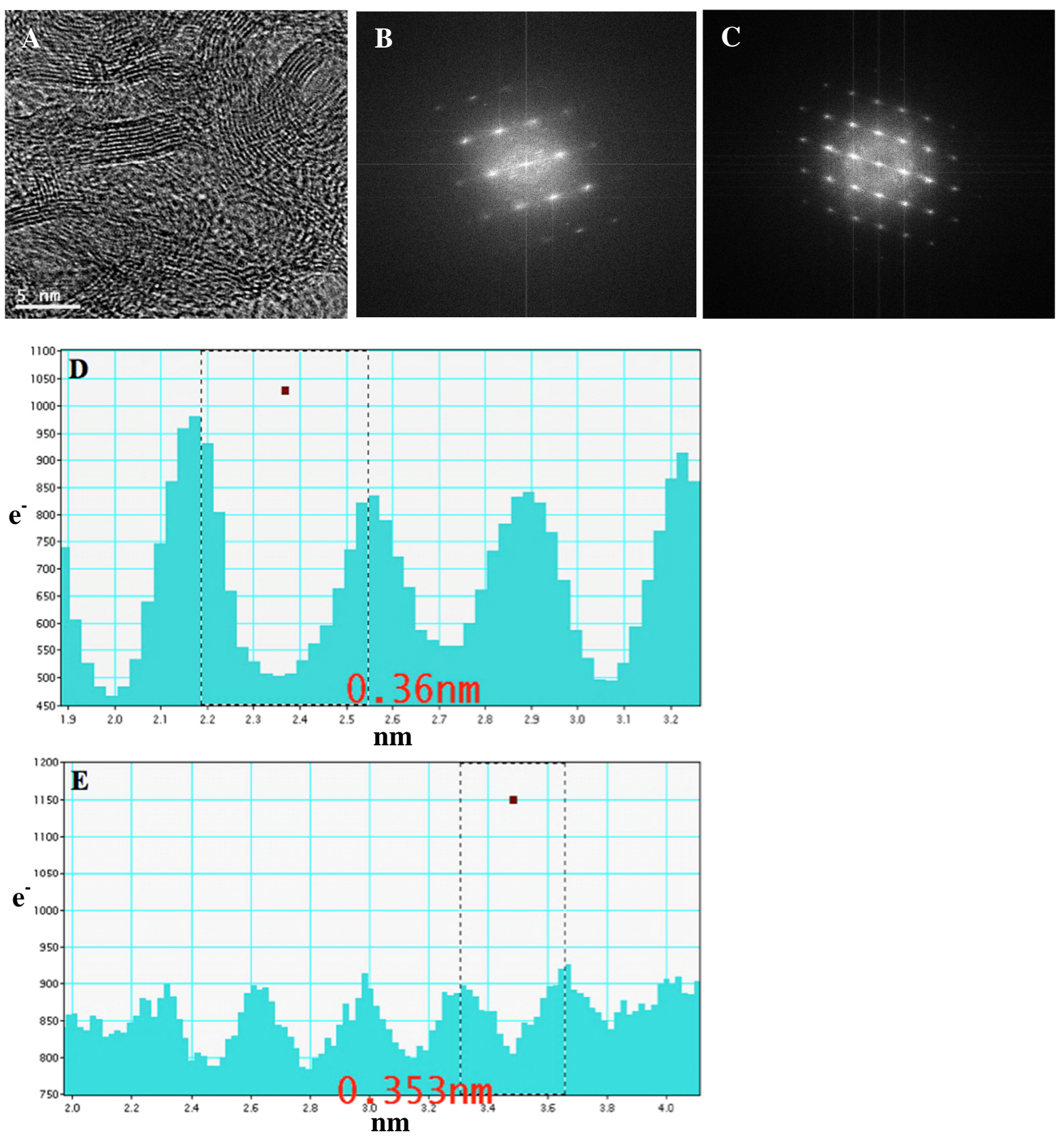

Figure 3(A): HRTEM micrograph, $(\mathrm{B}, \mathrm{C})$ selective area electron diffraction (SAED) patterns, and (C-D) intensity profiles SU8 derive glassy carbon pyrolyzed at $900^{\circ} \mathrm{C}$. Images (B-E) were taken at two different graphene ribbon positions for the HRTEM micrograph shown in (A).

$\underline{\text { Nanoindentation }}$

A typical nanoindentation force-displacement curve for a $\sim 10 \mu \mathrm{m}$ thick SU8 derived GC film is shown in Figure 4(A). An average hardness of $1.877 \mathrm{GPa}$ and an average Young's modulus of 9.86 GPa were measured from this set of tests. Schueller et al. reported Young's modulus values 
between 10-40 GPa for polymer (furfuryl alcohol based resin) derived GC(21). The value for the Young's modulus we measure here is in that same range, although toward the low end of the reported values. Since SU8 is an epoxy based phenol-formaldehyde resin, some difference in the microstructure and therefore Young's modulus is expected.

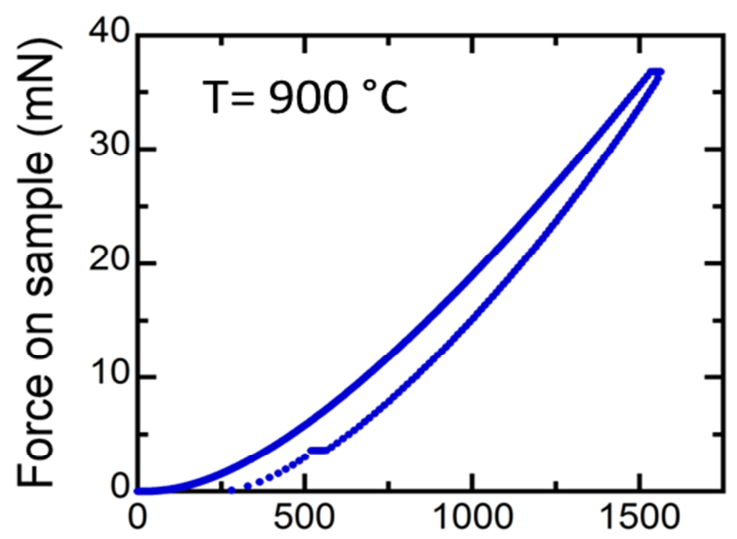

(A) Displacement into surface $(\mathrm{nm})$

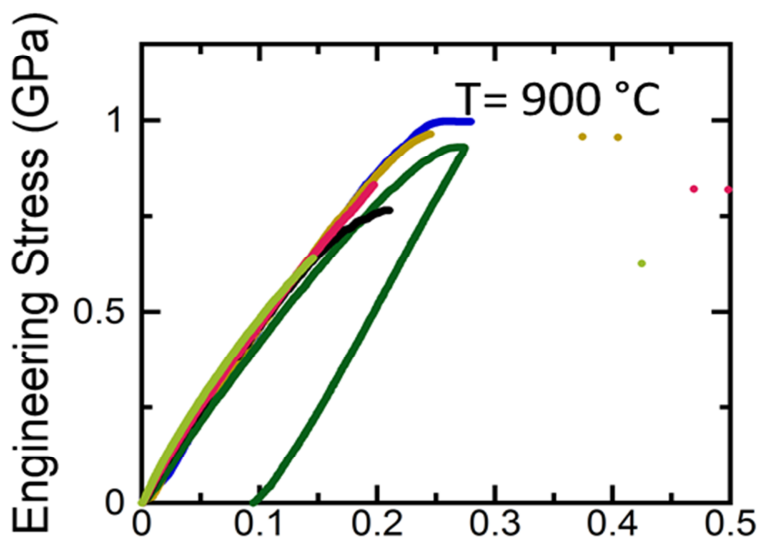

(B) Engineering Strain

Figure 4: Mechanical behavior of (A) SU8 derived glassy carbon film on nanoindentation, (B) SU8 derived glassy carbon micropillars on microcompression.

The microcompression tests were carried out on 5 specimens chosen from a large array of micropillars. The engineering stress-strain curves of these pillars are shown in Figure 4(B). The average strength of the micropillars was $0.852 \mathrm{GPa}$ with a standard deviation of $0.122 \mathrm{GPa}$. To the best of our knowledge, the microcompression tests on GC shown here are the first of their kind; microcompression tests on GC micropillars obtained from pyrolysis of any organic polymer have not been reported elsewhere.

As can be observed from Figure 4(B), during microcompression, there is a deviation from linear elasticity and a clear change to plastic deformation with limited plastic strain. This behavior reflects the brittle nature of the pillars annealed at $900^{\circ} \mathrm{C}$. At the end of the loading cycle, each pillar broke near the substrate attachment point and was ejected away. When this happens, the indenter tip traveled down quickly and hit the substrate, since the nanoindentation system is inherently load-controlled. This behavior was confirmed with optical microscopy after each test. This behavior is likely caused by either (i) a slight taper close to the bottom of the pillars where the highest stress builds and causes fracture, or (ii) poor adhesion of the pillars onto the $\mathrm{Si}$ substrate.

Some of the pillars failed before exhibiting clear yield behavior. A few of the stress-strain curves in Figure 4(B) show failure before any deviation from linear elasticity is detected. This behavior may be due to differences in the geometry of the individual pillars or variation in adhesion strength. In principle, one can fabricate almost identical pillars in a single array employing photolithography. However, during mechanical tests even a minor geometric or adhesion difference can lead to a significantly different maximum achievable stress. These 
results also demonstrate that, during battery operation, the weak mechanical point might well be between the CMEMS GC and the substrate.

\section{Conclusions and Future Work}

The larger graphene interlayer separation of GC fabricated using CMEMS/CNEMS compared to that in crystalline graphite, the feasibility of fabricating pre-defined high surface area electrode designs of any shape on a variety of substrates, and the excellent thermal and mechanical properties render this material and fabrication method very suitable for novel high energy density $\mathrm{Li}$ ion batteries. CMEMS/CNEMS technology is scalable and compatible with batch manufacturing techniques of electrodes of any geometry including fractal-like electrodes featuring micro and nano features. Such fractal-like electrodes minimize internal resistance while maximizing surface-to-volume ratios.

In terms of mechanical properties, CMEMS pillars with good substrate adhesion show excellent intrinsic mechanical properties and break from the substrate only at the end of the loading cycle. Further improvement in adhesion could be obtained by, for example, hexamethyldisilane (HMDS) treatment of the substrate. Optimized photolithography and pyrolysis processes together with improved substrate adhesion could lead to micropillars that could improve Li ion batteries on a larger scale. The pyrolysis process could be further optimized to tune the microstructure of the resulting carbon to allow for yet higher reversible battery capacity. In addition to the two- and three-dimensional GC CMEMS structures described here, we are also exploring the mechanical properties of the structures pyrolyzed below $900^{\circ} \mathrm{C}$ in order to understand the pyrolysis mechanism better. The effect of temperature ramp rate on the microstructure of the resulting GC material, and the mechanical and electrical properties of the SU8- GC intermediate materials are also under investigation.

Other advanced research fields that can benefit from the CMEMS technology by exploiting the excellent electrochemical and electrical properties of SU8 derived GC include interdigitated electrode arrays (IDEAs), on-chip cell separation by dielectrophoresis (DEP), fuel cells and various chemical sensors (e.g., nano gas sensors) and biosensors.

\section{References}

1. M. J. Madou, Fundamentals of microfabrication: the science of miniaturization (CRC Press, Boca Raton, 2nd ed., 2002).

2. S. Sharma, A. Sharma, Y.-K. Cho, M. Madou, ACS Appl. Mater. Interfaces 4, 34-39 (2012).

3. K. Kinoshita, Carbon: electrochemical and physicochemical properties (Wiley, New York, 1988).

4. S. Sharma, M. Madou, Bioinspired Biomim. Nanobiomaterials 1, 252-265 (2012).

5. C. Wang et al., in H. Urey, A. El-Fatatry (Eds). Carbon-MEMS architectures for 3D microbatteries, 295-302 (2004).

6. K. Kinoshita, X. Song, J. Kim, M. Inaba, J. Power Sources 81-82, 170-175 (1999). 
7. R. E. García, Y.-M. Chiang, W. Craig Carter, P. Limthongkul, C. M. Bishop, J. Electrochem. Soc. 152, A255 (2005).

8. S. Flandrois, B. Simon, Carbon 37, 165-180 (1999).

9. C. Wang, R. Zaouk, B. Y. Park, M. J. Madou, Int. J. Manuf. Technol. Manag. 13, 360 (2008).

10. K. Malladi, C. Wang, M. Madou, Carbon 44, 2602-2607 (2006).

11. R. Martinez-Duarte, R. A. Gorkin III, K. Abi-Samra, M. J. Madou, Lab. Chip 10, 1030 (2010).

12. C. Wang, L. Taherabadi, G. Jia, M. Madou, Y. Yeh, B. Dunn, Electrochem. Solid-State Lett. 7, A435 (2004).

13. U. Ramamurty, J. Jang, CrystEngComm (2013), doi:10.1039/c3ce41266k.

14. B. J. Briscoe, L. Fiori, E. Pelillo, J. Phys. Appl. Phys. 31, 2395-2405 (1998).

15. S. Natarajan, D. A. Chang-Yen, B. K. Gale, J. Micromechanics Microengineering 18, 045021 (2008).

16. S. Sharma, M. Madou, Philos. Trans. R. Soc. Math. Phys. Eng. Sci. 370, 2448-2473 (2012).

17. S. Sharma, S. Borrós-Cullell, M. H. Asadian Ardakani, M. Madou, Microelectron. Eng. , Article under review.

18. F. Hofer, in Active Metals, A. Frstner, Ed. (Wiley-VCH Verlag GmbH, Weinheim, Germany), pp. 427-446.

19. P. J. F. Harris, Philos. Mag. 84, 3159-3167 (2004).

20. W. Xing, J. Electrochem. Soc. 143, 3482 (1996).

21. O. J. A. Schueller, S. T. Brittain, G. M. Whitesides, Adv. Mater. 9, 477-480 (1997). 